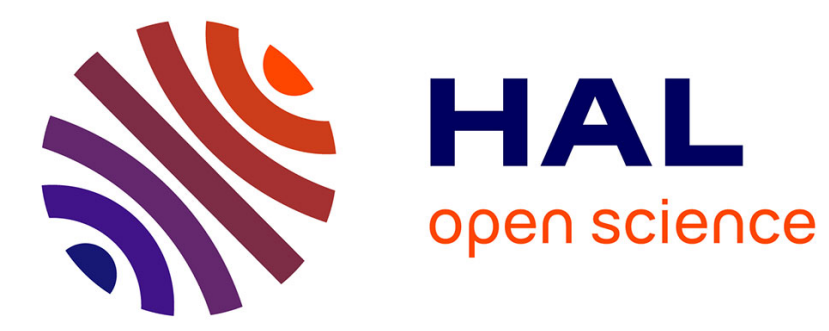

\title{
Conjugated Copolymers That Shouldn't Be
}

Jun Guan, Zejun Sun, Ramin Ansari, Yujia Liu, Aimi Endo, Masafumi Unno,

Armelle Ouali, Shahrea Mahbub, Joseph C Furgal, Nuttapon Yodsin, et al.

\section{To cite this version:}

Jun Guan, Zejun Sun, Ramin Ansari, Yujia Liu, Aimi Endo, et al.. Conjugated Copolymers That Shouldn't Be. Angewandte Chemie International Edition, 2021, 60 (20), pp.11115-11119. 10.1002/anie.202014932 . hal-03425753

\section{HAL Id: hal-03425753 \\ https://hal.science/hal-03425753}

Submitted on 11 Nov 2021

HAL is a multi-disciplinary open access archive for the deposit and dissemination of scientific research documents, whether they are published or not. The documents may come from teaching and research institutions in France or abroad, or from public or private research centers.
L'archive ouverte pluridisciplinaire HAL, est destinée au dépôt et à la diffusion de documents scientifiques de niveau recherche, publiés ou non, émanant des établissements d'enseignement et de recherche français ou étrangers, des laboratoires publics ou privés. 
DOI : $10.1002 /$ anie.202014932

Communication

\section{Conjugated Copolymers That Shouldn't Be}

Jun Guan 0000-0002-0860-2204,[a] Zejun Sun, [c] Ramin Ansari, [b] Yujia Liu 0000-0002-3610-6355, [d] Aimi Endo, [d] Masafumi Unno 0000-0003-2158-1644, [d] Armelle Ouali 0000-0001-7436-776X,[e] Shahrea Mahbub, [f] Joseph^^C. Furgal 0000-0002-7040-0793, [f] Nuttapon Yodsin, ${ }^{[g]}$ Siriporn Jungsuttiwong, ${ }^{[g]}$ Daniel Hashemi, ${ }^{[a]}$ John Kieffer 0000-0002-3108-2323,[a] Prof. Richard^^M. Laine $0000-0003-4939-3514 *[a]$

Unexpected excited-state conjugation was observed in a series of copolymers derived from ladder silsesquioxane, vinyl (Me/Ph) Si $\left(\mathrm{O}_{0.5}\right)_{2}\left[\mathrm{PhSiO}_{1.5}\right]_{4}\left(\mathrm{O}_{0.5}\right)_{2} \mathrm{Si}(\mathrm{Me} / \mathrm{Ph}) \mathrm{vinyl}, \quad$ which exhibited 30--60^^nm red-shifted emission relative to doubledecker derived analogues with equal or larger degrees of polymerization. Further studies hint at their potential application as semiconducting polymers.

\#\#

Semiconducting Copolymers

copolymerization

ladder

silsesquioxanes

polymers

semiconducting

materials

silsesquioxanes

\section{Abstract}

Multiple studies have explored using cage silsesquioxanes (SQS) as backbone elements in hybrid polymers motivated by 
their well-defined structures and physical and mechanical properties. As part of this general exploration, we report unexpected photophysical properties of copolymers derived from divinyl double

decker SQS, [vinyl (Me) Si (O..5) 2] [ $\left.\mathrm{PhSiO}_{1.5}\right]_{8}\left[\left(\mathrm{O}_{0.5}\right)_{2} \mathrm{Si}(\mathrm{Me})\right.$ vinyl] (vinyldDvinyl). These copolymers exhibit strong emission redshifts <?>relative to<?> model compounds, implying unconventional conjugation, despite vinyl(Me)Si(O-) 2 siloxane bridges. In an effort to identify minimum sQ structures that do/do not offer extended conjugation, we explored Heck catalyzed co-polymerization of vinyl-ladder(LL)-vinyl compounds, vinyl (Me/Ph)Si $\left(\mathrm{O}_{0.5}\right)_{2}\left[\mathrm{PhSiO}_{1.5}\right]_{4}\left(\mathrm{O}_{0.5}\right)_{2} \mathrm{Si}(\mathrm{Me} / \mathrm{Ph}) \mathrm{vinyl}$, with Br-Ar-Br. Most surprising, the resulting oligomers show 30--60^^nm emission red-shifts beyond those seen with vinyldDvinyl analogs despite lacking a true cage. Further evidence for unconventional conjugation includes apparent integer charge transfer (ICT) between LL-co-thiophene, bithiophene, and thienothiophene with 10^^mol^o $\mathrm{F}_{4} \mathrm{TCNQ}$, suggesting potential as p-type doped organic/inorganic semiconductors.

\section{Main text}

There is growing impetus to explore silsesquioxanes (SQS) due to their ease of synthesis and purification, well-defined 3D nanostructures, robust nature as well as high compatibility with multiple types of organics leading to large families of hybrid materials. The major story still centers on properties that can be typified as organic decorated silica moieties. In contrast, we find that cage SQs $\left\{\left[\mathrm{RSiO}_{1.5}\right]_{8,10,12}\right.$ (R=R'phenyl, R'vinyl, $\mathrm{R}=$ conjugated moiety, $\mathrm{R}^{\prime}=\mathrm{H}, \mathrm{Me}, \mathrm{MeO}, \mathrm{NH}_{2}, \mathrm{CN}$, etc.) \} 
offer photophysical properties not at all reflective of organic decorated silicas but commensurate with cage centered LUMOs. ${ }^{[1--5]}$ We recently extended this concept of excited-state 3D conjugation to sets of incomplete and modified cages including corner-missing $\mathrm{T}_{8}, \quad$ [R'StilbeneSiO $\left.{ }_{1.5}\right]_{7}\left[\mathrm{O}_{1.5} \mathrm{SiMe/nPr}\right.$, [R'StilbeneSiO $\left.{ }_{1.5}\right]_{7}\left[\mathrm{O}_{0.5} \mathrm{SiMe}_{3}\right]_{3}{ }^{[6]}$ and newly developed double decker

[R'StilbeneSiOn.5] $8\left[\mathrm{OSiMe}_{2}\right]_{2}$

and [R'StilbeneSiO $\left.{ }_{1.5}\right]_{8}\left[\mathrm{O}_{0.5} \mathrm{SiMe}_{3}\right]_{4} \cdot{ }^{[7]}$ Recent efforts on double decker sos follow two parallel paths in their development exploring either molecular or macromolecular hybrid materials. ${ }^{[8]}$ In the latter area, we recently synthesized a series of DD $S Q$ based oligomers and polymers \{vinyl (Me) Si (O..5) $2\left[\mathrm{PhSiO}_{1.5}\right]_{8}\left(\mathrm{O}_{0.5}\right)_{2} \mathrm{Si}(\mathrm{Me})$ vinyl-Ar, vinyldDvinylAr\} that unexpectedly exhibit conjugation that appears to involve two vinyl(Me)Si(O-) 2 bridges evidenced by significant emission red-shifts from corresponding model compounds. [9] Schemes^^1^a<schr1>,b provide general synthetic routes and structures. In all systems, conjugation presents in the form of emission red-shifts of 50--120^^nm from model compounds without cage components. Such novel combinations of structural, thermal, mechanical and photophysical properties, such as tunable broad-band UV/Vis fluorescence, may be important for applications as emissive layers in OLEDs, [10] multi-functional nanodrugs, in photothermal and photodynamic therapies for cancer, etc. ${ }^{[11--13]}$

With the recent discovery of routes to ladder sQs (vinylLL-vinyl), [14] we can now explore vinyl-LL-vinyl equivalent systems of the type suggested in Scheme^^1^c<xschr1>. The motivation is to ascertain the nature of LUMO formation in ladder SQs where the structure appears to be approximately 
one-half that of relatively complete double-deckers. Our initial presumption was that these compounds should represent the limiting case where not only is there no 3D cage, but there are also two vinyl (Me/Ph)Si(O-)2 bridges per cage in any oligomers/polymers we might synthesize. Contrary to our original thoughts, the resulting compounds actually offer emission further red-shifted than any analogous compounds previously studied, ${ }^{[1--7, \wedge 9]}$ seeming to imply conjugation and semiconducting behavior where there should be none. In the following sections, we begin by characterizing a set of ladder copolymers and thereafter assess their photophysical properties.

Two structurally similar ladder SQS [vinyl-(Me)LL(Me)vinyl and vinyl-(Ph)LL(Ph)-vinyl] were explored, with only the end groups differing. Analytical characterization and photophysical behavior are essentially identical as expected, thus only data for vinyl-LL(Me)-vinyl co-polymers are presented. Spectra for vinyl-LL(Ph)-vinyl derived copolymers are shown in Figures^^s9--14. Both co-polymers were characterized by MALDI-TOF, GPC, FTIR, ${ }^{1} \mathrm{H}$ and ${ }^{13} \mathrm{C}^{\wedge} \wedge \mathrm{NMR}$, and the starting ladder ses were also characterized by ${ }^{29} \mathrm{Si}$ NMR as recorded in Tables^^S1--3 and Figures^^S1--8. MALDI-TOF shows the expected $\mathrm{m} / \mathrm{z}$, consistent with GPC determined molar masses of the ladder SQS. The GPC retention time for vinyl-LL(Ph)vinyl is $\approx 0.3^{\wedge} \wedge$ min earlier than that for vinyl-LL(Me)-vinyl due to the slightly bulkier phenyl groups. Table^^ $1<$ tabrl> GPC data for the ladder copolymers indicates degrees of polymerization (DPS) of 3--18 units. MALDI-TOF analyses always show peaks every $\mathrm{m} / \mathrm{z}$ for the co-monomer unit vinyl-LL-vinyl- 
Ar, suggesting no di-substitution of aromatic groups on any given vinyl and successful copolymerization.

The steady-state absorption and emission behavior of ladder polymers are shown in Table^^ $1<x t a b r 1>$, Figure^^1<figrl> and Figure^^2<figr2>, compared with data for vinylDDvinyl derived polymers from our previous study. [9] All emission spectra were measured with excitation wavelength at corresponding absorption $\lambda_{\max }$. The Table^^1<xtabr $1>$ data for vinyl(Me)DD(Me)vinyl and vinyl-LL(Me)-vinyl indicates that all exhibit absorption $\lambda_{\max } \approx 265^{\wedge} \wedge \mathrm{nm}$ and emission $\lambda_{\max } \approx 280^{\wedge} \wedge \mathrm{nm}$, typical for phenyl rings. Vinyl-LL(Ph)-vinyl is slightly redshifted with phenyls at two ends. Polymers were synthesized from both vinyl-LL-vinyl compounds. No essential spectral differences were observed as expected.

Divinylbenzene is the simplest organic linker tested. The steady-state data in Table^^1<xtabrl> for LL-co-phenyl are essentially identical to those of DD-co-phenyl. The red shift of $70^{\wedge \wedge} \mathrm{nm}$ in emission compared to 1,4-[(MeO) ${ }_{2}$ Sivinyl] ${ }_{2}$ benzene suggests formation of a LUMO even with a half cage and conjugation to co-phenyl in the excited state. Based on all our previous work, it appears that a LUMO does form and these results greatly extend the families of SQs that appear to offer extended conjugation. Even though the shifts are not significant in the absorption and emission $\lambda_{\max }$ of LL-co-phenyl from its DD analogue, there are significant changes in the shape of steady-state spectra as seen in Figure^^1<xfigr $1>$. First, LL-co-phenyl with a DP of 8 displays distinct absorption shoulders around $35^{\wedge \wedge}$ nm compared to model compound and DD-Co-phenyl with a DP of 15. Additionally, its emission 
is slightly red-shifted beyond DD-co-phenyl, along with the disappearance of the emission peak near $340^{\wedge} \wedge \mathrm{nm}$ and a significant shoulder at $\approx 450^{\wedge \wedge} \mathrm{nm}$.

Such changes in steady-state behavior are more apparent in LL-co-biphenyl, terphenyl, stilbene and thiophene as witnessed by $30--60^{\wedge}$ nm red-shifts beyond their DD analogues even with shorter chains, again suggesting LUMO formation even in ladder structures but also more efficient communication between the ladder SQs and the conjugated organic moieties in the excited state as compared to full and double-decker sQs. This finding is quite important in that it completely changes our assumptions about ladder SQs. To further probe such structureproperty relationships, a shorter fraction of LL-co-thiophene was separated via TLC (silica, 1:1 DCM:hexane) and characterized by GPC and MALDI-TOF in Figure^^s15 and Table^^ $2<$ tabr $2>$.

The GPC of the starting vinyl-LL-vinyl, short LL-cothiophene separated by TLC (DP $\approx 3)$ and long LL-co-thiophene $(\mathrm{DP} \approx 7)$ are shown in Figure^^S15. The GPC trace of the LL-Cothiophene with $\mathrm{DP} \approx 7$ shows a broad peak around $27.5^{\wedge \wedge} \mathrm{min}$ with a $\boxplus$ of 1.43 for relatively long oligomers as well as a quite narrow peak at $33.2^{\wedge \wedge}$ min overlapping with vinyl-LL-vinyl with polydispersity $(\boxplus)$ of 1.02 , suggesting presence of unreacted starting material during copolymerization. The short LL-cothiophene with $\mathrm{DP} \approx 3$ comes at $31.5^{\wedge}$ min with a small $\boxplus$ of 1.12 , dominated by dimers and trimers following removal of longer oligomers via TLC. The GPC and MALDI-TOF data are shown in Table^^2<xtabr2>. The largest oligomers identifiable by MALDITOF with corresponding masses are presented. It is known that 
the peak intensities in MALDI corresponds to the ionization efficiencies of the species and are not necessarily representative of the quantity of each component. High $\mathrm{M}_{\mathrm{w}}$ oligomers are not as readily ionizable as monomers thus even though the GPC shows even higher $\mathrm{M}_{\mathrm{w}}$ oligomers, they are not necessarily "seen" in MALDI. Data for DD-co-thiophene with DP $\approx 20$ are also presented here. Since LL-co-thiophene is shorter than its DD analogue, it is safe to say that its red-shifted emission comes from more efficient electronic communication between LL SQs in the excited state rather than longer chains with extended conjugation lengths. extend the families of SQS that appear to offer extended conjugation.

Figure^^2<xfigr2> presents the normalized steady-state emission spectra for 2,5-[(MeO) ${ }_{2}$ Sivinyl]2thiophene, DD-Cothiophene, LL-co-thiophene with DPs of 3 and 7 respectively. The absorption $\lambda_{\max }$ is always around $340^{\wedge \wedge} \mathrm{nm}$ and not shown here. The progressively red-shifted emission $\lambda_{\max }$ of short and long LL-co-thiophenes are 484 and 539^^nm, respectively, suggesting extended conjugation with further extensions in chain length and smaller band gaps. Short LL-co-thiophene, consisting mostly of dimers and trimers, still shows $\lambda_{\max }$ emission. similar to long DD-co-thiophene, which is $\approx 50^{\wedge} \wedge \mathrm{nm}$ red-shifted from 2,5-[(MeO) ${ }_{2}$ Sivinyl] 2 thiophene. It has been reported that $\mathrm{T}_{8} \mathrm{SQ}$ cages exhibit electron-withdrawing capacities approximately equivalent to $-\mathrm{CF}_{3} \cdot{ }^{[15]}$ One can extend this idea to DD SQs given similar structures and presumably LL systems. It then becomes possible to argue that DD-cothiophene oligomers and even dimers and trimers of LL-cothiophene should offer similar properties. This further confirms the efficient semiconducting behavior of such ladder 
SQs polymers. Similar study has also done on LL-co-biphenyl as an example from the phenyl system of LL copolymers and the GPC and steady-state emission spectra are shown in Figure^^s16, 17. The progressive red-shifts in the emission of LL-cobiphenyl as a function of DPs have also been observed.

Further evidence of unconventional conjugation includes the charge-transfer studies of thiophene systems of LL SQ copolymers. Integer charge transfer (ICT) is observed between electron-acceptor $2,3,5,6$-tetrafluoro-7, 7,8,8tetracyanoquinodi-methane ( $\left.\mathrm{F}_{4} \mathrm{TCNQ}\right)$ and DD-Co-thiophene, bithiophene and thienothiophene as reported in our previous paper. ${ }^{[9]}$ Similar ICT also occurred in 10^^mol^^${ }^{\wedge} \mathrm{F}_{4} \mathrm{TCNQ}$ doped LLco-thiophene, bithiophene and thienothiophene using the mixedsolution method. The original orange-red color becomes dark green immediately on mixing. The literature reports that poly(3-hexyl-thiophene-2,5-diyl) regioregular (P3HT) exhibits integer charge transfer interactions with $\mathrm{F}_{4} \mathrm{TCNQ}$ and coincident nitrile-stretching mode shifts from a neutral value $\nu_{0}=2227^{\wedge} \mathrm{Cm}^{<\mathrm{M}->1}$ to $\nu_{1}=2194^{\wedge}{ }^{\wedge} \mathrm{Cm}^{<\mathrm{M}->1}$ on doping. ${ }^{[16, \wedge 17]}$ In marked contrast, however, quaterthiophene (4T) forms partial charge transfer with $F_{4}$ TCNQ and thus shows only a small shift. [18] Shifts of characteristic cyano-vibrational bands from the neutral value to the anion value are observed in the FTIR for LL-Co-bithiophene mixing with F $_{4}$ TCNQ as shown in Figure^^3<figr3> indicating integer charge transfer (ICT) .

The literature also reports that the electronic structure of $\mathrm{F}_{4} \mathrm{TCN}$ anion includes doublet states because of the presence of an unpaired single electron. ${ }^{[19]}$ The UV/Vis spectrum of $\mathrm{F}_{4} \mathrm{TCNQ}^{\cdot<\mathrm{M}->}$ contains two main absorption peaks around 400 and 
800^^nm. The $\mathrm{D}_{0} \rightarrow \mathrm{D}_{1}$ transition corresponds to the absorption band around 600--900^^nm with local maxima at 754 and $856^{\wedge \wedge}$ nm while the $\mathrm{D}_{0} \rightarrow \mathrm{D}_{2}$ transition gives absorption band at $410^{\wedge} \wedge \mathrm{nm}$. The absorption spectrum of $\mathrm{LL}(\mathrm{Ph})$-co-bithiophene: $\mathrm{F}_{4} \mathrm{TCN}$ in Figure^^4<figr4> clearly shows spectral signatures from $\mathrm{F}_{4} \mathrm{TCNQ}^{\cdot<\mathrm{M}->}$ at $600--900^{\wedge} \mathrm{nm}$ and LL-Co-bithiophene at 350-500^^nm, strongly suggesting ICT, consistent with the FTIR data.

Further evidence of unconventional conjugation comes from breaking and restoring conjugation by brominating/debrominating vinyls. Our original objective in brominating phenyl groups in DD/LL-Co-phenyl polymers was to further functionalize the phenyl groups peripherally to prepare "hairy polymers" and explore possible 3D conjugation. As expected, vinyl groups brominate first as evidenced by Figure^^S16--19. ${ }^{13} \mathrm{C}$ and ${ }^{29} \mathrm{Si}$ NMR coincident with elimination of conjugation and partial cage cleavage as tracked by GPC. As shown in

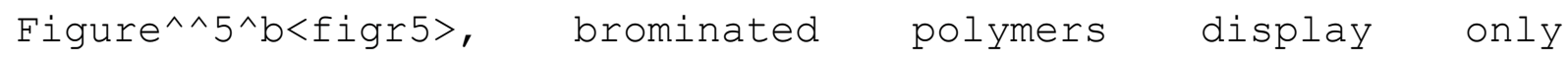
characteristic phenyl absorption around 250^^nm. The emission spectra are diminished and only show $\mathrm{CH}_{2} \mathrm{Cl}_{2}$ solvent background. The photophysical behavior of both DD/LL-co-phenyl reappear after zinc debromination, ${ }^{[20]}$ with absorption $\lambda_{\max }$ around $300^{\wedge} \wedge \mathrm{nm}$ and red-shifted emission $\lambda_{\max }$ around $400^{\wedge} \wedge \mathrm{nm}$ with respect to the emission of 1,4-[(MeO) ${ }_{2}$ Sivinyl] ${ }_{2}$ benzene at $332^{\wedge} \wedge n m$. This finding further proves the presence and reproducibility of the excited-state conjugation in the DD/LL SQ derived polymers via vinylsi(O-) 2 siloxane unit.

Two modeling groups attempted to model the vinyl-LL-vinyl SQ structure. Professor Jungsuttiwong's group at Ubon 
Ratchathani University, Thailand, and Professor Kieffer's group at the University of Michigan, found both HOMOs and LUMOs reside on the peripheral phenyl groups of vinylLL (Me/Ph)-vinyl, with an energy band gap of $\approx 8.9^{\wedge} \mathrm{eV}^{\mathrm{N}}$. The calculated absorption $\lambda_{\max }$ is always $\approx 60^{\wedge} \wedge \mathrm{nm}$ blue-shifted compared to the experimental data, as shown in Table^^s4. In another effort from Professor Kieffer's group the peripheral phenyl groups are replaced by methyl for modeling purposes, LUMO+2 at $8.47^{\wedge} \wedge \mathrm{eV}$ is from LL SQ core and extends out of the ladder frame, suggesting possible interaction between an SQcentered LUMO and vinyl $\Pi^{*}$. Orbitals. When phenyls are retained, the LUMO+10 also resides in the center of LL SQ and extends out to vinyl $\Pi^{*}$ with a lower energy level at $7.86^{\wedge} \mathrm{eV}$. These modeling results are presented in Figure^^s26--28. At this time, modeling is still not as precise as we would like but these systems are quite unique as our results demonstrate.

In summary, a series of ladder (LL) SQ copolymers were synthesized via Heck catalytic cross-coupling of vinyl-LLvinyl with various dibromo-aromatic compounds. Compared to model silane compounds and analogs of double-decker (DD) SQ copolymers, LL derived polymers display similar absorption peaks but with significant shoulders at longer wavelength. Furthermore, LL SQs linked with longer or more complex aromatic bridges, LL-co-biphenyl, terphenyl, stilbene and thiophene, show 30--60^^nm emission red-shifts beyond those seen with the DD analogs and progressively red-shifted emissions with respect to longer chains were also observed. Integral charge transfer occurs in 10^^mol^응 $\mathrm{F}_{4} \mathrm{TCNQ}$-doped LLco-thiophene, bithiophene, thienothiophene, which also occur on mixing $\mathrm{F}_{4} \mathrm{TCN}$ with $\mathrm{DD}$ analogous polymers. These results 
suggest not only a new perspective on the ease of formation of LUMOS in even LL SQS, but also more efficient communication of the LL SQ with conjugated organic moieties in the excited state as compared to full and double-decker sQs that potentially offer access to a wide variety of semiconducting polymers. In addition, brominating vinyl as well as phenyl groups in DD/LL derived copolymers and then debrominating vinyls not only restores through chain conjugation but also provides the potential to further peripherally functionalize these systems via a variety of catalytic cross-coupling reactions to explore 3D conjugation in "hairy SQ polymers".

\section{Acknowledgements}

We thank NSF for support of this work through Chem. Award No. 1610344. J.C.F. thanks Bowling Green State University for Startup Funding. S.J. thanks the Center of Excellence for Innovation in Chemistry (PERCH-CIC), Ministry of Higher Education, Science, Research and Innovation, the Human

Resources Development in Science Project Science Achievement Scholarship of Thailand (SAST) for support. J.K. thanks NSFDMR-1435965 for support.

\section{Conflict of interest}

The authors declare no conflict of interest.

\section{Author Contributions}

J.G. Conceptualization: Equal; Data curation: Equal; Formal analysis: Equal; Writing----original draft: Equal; Writing---review \&amp; editing: Equal. 
<litl $><j n l>J \cdot{ }^{\wedge}{ }^{\wedge}$. Furgal, J.^^ $\mathrm{H}$. Jung, T. Goodson, R. ${ }^{\wedge} \mathrm{M}$. Laine, J. Am. Chem. Soc. 2013, 135, 12259--12269</jnl>.

<lit2><jnl>R.^^M. Laine, S. Sulaiman, C. Brick, M. Roll, R. Tamaki, M.^^Z. Asuncion, M. Neurock, J.-S. Filhol, C.-Y. Lee, J. Zhang, T. Goodson, M. Ronchi, M. Pizzotti, S.^^C. Rand, Y. Li, J. Am. Chem. Soc. 2010, 132, 3708--3722</jnl>. <lit3><jnl>M.^^z. Asuncion, R.^^M. Laine, J. Am. Chem. Soc. 2010, 132, 3723--3736</jnl>.

<lit4><jnl>S. Sulaiman, J. Zhang, T. Goodson^^III, R.^^M. Laine, J. Mater. Chem. 2011, 21, 11177</jnl>.

<lit5><jnl>S. Sulaiman, A. Bhaskar, J. Zhang, R. Guda, T. Goodson, R.^^M. Laine, Chem. Mater. 2008, 20, 5563-$5573</ j n l>$.

<lit6><jnl>J. Guan, K. Tomobe, I. Madu, T. Goodson, K. Makhal, M.^^T. Trinh, S.^^C. Rand, N. Yodsin, S. Jungsuttiwong, R.^^M. Laine, Macromolecules 2019, 52, 4008--4019</jnl>.

<lit7 $><j n l>$ J. Guan, K. Tomobe, I. Madu, T. Goodson, K. Makhal, M.^^T. Trinh, S.^^C. Rand, N. Yodsin, S. Jungsuttiwong, R.^^M. Laine, Macromolecules 2019, 52, 7413--7422</jnl>.

<lit8><jnl>B. Dudziec, B. Marciniec, Curr. Org. Chem. 2017, 21, $2794--2813</ j n 1>$.

<lit9><jnl>J. Guan, J.^^J.^^R. Arias, K. Tomobe, R. Ansari,

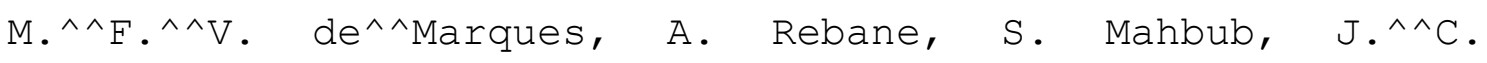
Furgal, N. Yodsin, S. Jungsuttiwong, D. Hashemi, J. Kieffer, R.^^M. Laine, ACS Appl. Polym. Mater. 2020, 2, $3894--3907</ j n l>$.

<litlo><jnl>K.^^L. Chan, P. Sonar, A. Sellinger, J. Mater. Chem. 2009, 19, 9103</jnl>. 
<lit11><jnl>Y. Cai, D. Ni, W. Cheng, C. Ji, Y. Wang, K. Müllen, Z. Su, Y. Liu, C. Chen, M. Yin, Angew. Chem. Int. Ed. 2020，59, 14014--14018; Angew. Chem. 2020, 132, 14118-$14122</ j n l>$.

<lit12><jnl>C. Ji, Q. Gao, X. Dong, W. Yin, Z. Gu, Z. Gan, Y. Zhao, M. Yin, Angew. Chem. Int. Ed. 2018, 57, 11384--11388; Angew. Chem. 2018, 130, 11554--11558</jnl>.

<lit13><jnl>C. Liu, S. Zhang, J. Li, J. Wei, K. Müllen, M. Yin, Angew. Chem. Int. Ed. 2019, 58, 1638--1642; Angew. Chem. 2019, 131, 1652--1656</jnl>.

<lit14><jnl>Y. Liu, K. Onodera, N. Takeda, A. Ouali, M. Unno, Organometallics 2019, 38, 4373--4376</jnl>.

<lit15><jnl>F.^^J. Feher, T.^^A. Budzichowski, J. Organomet. Chem. 1989, 379, 33--40</jnl $>$.

<lit16><jnl>H. Méndez, G. Heimel, S. Winkler, J. Frisch, A. Opitz, K. Sauer, B. Wegner, M. Oehzelt, C. Röthel, S. Duhm, D. Többens, N. Koch, I. Salzmann, Nat. Commun. 2015, 6, $8560</ j n l>$.

<lit17><jnl>I.^^E. Jacobs, A.^^J. Moulé, Adv. Mater. 2017, 29, $1703063</ j n l>$.

<lit18><jnl>I. Salzmann, G. Heimel, M. Oehzelt, S. Winkler, N. Koch, AcC. Chem. Res. 2016, 49, 370--378</jnl>.

<lit19><jnl>L. Ma, P. Hu, H. Jiang, C. Kloc, H. Sun, C. Soci, A.^^A. Voityuk, M.^^E. Michel-Beyerle, G.^^G. Gurzadyan, SCi. Rep. 2016, 6, $28510</ j n 1>$.

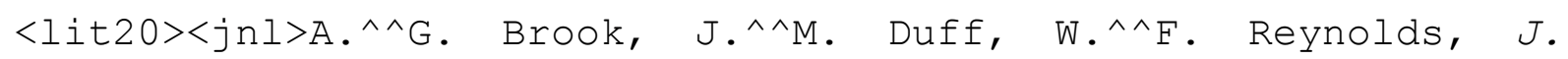
Organomet. Chem. 1976, 121, 293--306</jnl>. 


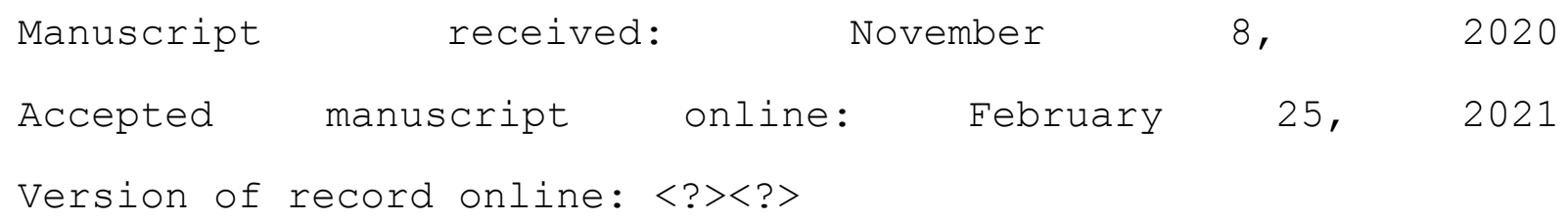

Scheme^^1 Heck cross-coupling of a)^^model compounds, b)^^vinyldDvinyl derived copolymers and c)^^vinyl-LL-vinyl derived copolymers.

Figure^^1 Normalized steady-state absorption and emission spectra of 1,4-[(MeO) ${ }_{2}$ Sivinyl] ${ }_{2}$ benzene, DD-co-phenyl and LL-Cophenyl in $\mathrm{CH}_{2} \mathrm{Cl}_{2}$.

Figure^^2 Normalized progressive emission spectra of 2,5[(MeO) ${ }_{2}$ Sivinyl] ${ }_{2}$ thiophene, DD-co-thiophene, short (DP $\left.\approx 3\right)$ and long $(\mathrm{DP} \approx 7)$ LL-Co-thiophene in $\mathrm{CH}_{2} \mathrm{Cl}_{2}$.

Figure^^3 $v$ CN region in the FTIR spectra of pristine $\mathrm{F}_{4} \mathrm{TCN}$ and a mixture of LL-CO-bithiophene: $\mathrm{F}_{4} \mathrm{TCNQ} 10^{\wedge} \mathrm{mol}^{\wedge} \%$.

Figure^^4 Absorption spectra of undoped LL-co-bithiophene and LL-Co-bithiophene; $\mathrm{F}_{4} \mathrm{TCNQ} 10^{\wedge} \mathrm{mol}^{\wedge} \circ, \mathrm{F}_{4} \mathrm{TCNQ}$ and $\mathrm{F}_{4} \mathrm{TCNQ}{ }^{\cdot<\mathrm{M}->}$ shown for reference.

Figure^^5 a)^^Bromination and debromination of LL-co-phenyl. b)^^Steady-state absorption and emission spectra in $\mathrm{CH}_{2} \mathrm{Cl}_{2}$.

Table^^1 GPC and steady-state photophysical data for vinylDDvinyl derived polymers ${ }^{9}$ and vinyl-LL(Me)-vinyl derived polymers.

$\mathrm{DP}^{[\mathrm{a}]} \mathrm{Abs} \cdot \lambda_{\max }$ Em. $\lambda_{\max } \Phi_{\mathrm{F}}$

$\underline{[n m]} \quad[n]^{[b]}$

Vinyl (Me) DD (Me) vinyl $\quad 1 \quad 264 \quad 281$ 


\begin{tabular}{|c|c|c|c|c|}
\hline DD-co-phenyl & 15 & 298 & 390,412 & $0.08 \pm 0.001$ \\
\hline DD-co-biphenyl & 10 & 314 & 357,373 & $0.66 \pm 0.05$ \\
\hline DD-co-terphenyl & 11 & 321 & 374,392 & $0.87 \pm 0.04$ \\
\hline DD-co-stilbene & 7 & 357 & 412,436 & $0.61 \pm 0.04$ \\
\hline Co-dimethylfluorene & 15 & 339,353 & 424,448 & $0.34 \pm 0.003$ \\
\hline DD-co-thiophene & 20 & 340 & 478,505 & $0.09 \pm 0.001$ \\
\hline DD-co-bithiophene & 3 & 391 & 505,538 & $0.17 \pm 0.02$ \\
\hline Co-thienothiophene & 4 & 358 & 496,526 & $0.13 \pm 0.01$ \\
\hline Vinyl-LL (Me/Ph) -vinyl & 1 & 264 & 283 & \\
\hline LL-co-phenyl & 8 & 298 & 392,415 & $0.16 \pm 0.02$ \\
\hline LL-co-biphenyl & 6 & 312 & 412,430 & $0.46 \pm 0.04$ \\
\hline LL-co-terphenyl & 18 & 321 & 418,437 & $0.61 \pm 0.02$ \\
\hline LL-co-stilbene & 8 & 356 & $448, \quad 472$ & $0.35 \pm 0.03$ \\
\hline Co-dimethylfluorene & 7 & 337,353 & 426,451 & $0.68 \pm 0.02$ \\
\hline LL-co-thiophene & 7 & 343 & 540 & $0.07 \pm 0.01$ \\
\hline LL-co-bithiophene & 6 & 392 & 550 & $0.07 \pm 0.01$ \\
\hline Co-thienothiophene & 6 & 356,371 & 530 & $0.09 \pm 0.01$ \\
\hline
\end{tabular}

[a]^^Degree of polymerization. [b]^^Excitation wavelength at Abs. $\lambda_{\max }$. 
Table^^2 MALDI-TOF and GPC data for DD-Co-thiophene, short LLco-thiophene isolated by TLC and long LL-co-thiophene.

GPC

$\mathrm{M}_{\mathrm{n}}$

2540

5600

8000

$22^{\wedge} 540$

$43^{\wedge} 250$

1.92

decamer

$13^{\wedge} 010^{[\mathrm{c}]}$

$\mathrm{DP} \approx 20$

[a]^^Polydispersity. [b]^^Largest oligomers identifiable. $[\mathrm{c}]^{\wedge \wedge} \mathrm{As} \mathrm{Ag}^{+}$adduct . 